\title{
Limit of Low Temperatures, Freeze Decay, Dissipation of Matter, Harvest of Cold Energy
}

\author{
Jitendra Kumar Barthakur \\ Department of Statistics, Nutech Mediworld, Green Park Extension, New Delhi, India
}

Email address:

jkbarthakur@bol.net.in

To cite this article:

Jitendra Kumar Barthakur. Limit of Low Temperatures, Freeze Decay, Dissipation of Matter, Harvest of Cold Energy. American Journal of Modern Physics. Vol. 4, No. 5, 2015, pp. 217-220. doi: 10.11648/j.ajmp.20150405.11

\begin{abstract}
Background: The gas laws assert that at "absolute zero" or $0 \mathrm{~K}$, the gaseousness of the gases ends. Experimentally, gases become liquid or solid at $0 \mathrm{~K}$. However, (i) $0 \mathrm{~K}$ is not the lowest limit of temperature; and (ii) at $0 \mathrm{~K}$, the value of entropy is not zero. Activity continues at $0 \mathrm{~K}$. There is (a) "Zero-point" energy; (b) the principle of "indeterminacy" holds; (c) $0 \mathrm{~K}$ is not the lower limit of "critical points" of the fluids and solids phases; (d) the structures of atoms comport in "clusters"; (e) electronic energy "mismatch" in superconductivity; and other phenomena stand against zero entropy at 0K. Purpose: Dissipation of matter means dissipation of energy. It may be possible to harvest the dissipating energy at below $0 \mathrm{~K}$ temperature for the use of human beings. Method: A number of statistical equations anchor at zero entropy at $0 \mathrm{~K}$. This anchor is arbitrary. At $0 \mathrm{~K}$, gas stays as liquid, solid or plasma. Argumentatively, much below that temperature there is liquid-limittemperature (LLT) where all liquids become solids or plasma. Below LLT, there is solid-limit-temperature (SLT) where freeze decay of matter sets in. At SLT, the articles and sub-particles constituting an atom dance away in to space. Result: Matter exists in the range between very cold temperature and very hot temperature. When matter dissipates then energy disperses with the dissipating matter. Conclusion: Laboratories need to reach the SLT temperature empirically and find means to harvest the dissipating energy.
\end{abstract}

Keywords: Gas-Limit-Temperature, Liquid-Limit-Temperature, Solid-Limit-Temperature, Dissipation of Matter, Freeze-Decay

\section{Introduction}

Heat is foundational to understanding nature. This paper discusses about the low temperature.

Absolute zero of temperature, $0 \mathrm{~K}$, is the supposition of study of gases in physical chemistry. Boyle's Law (1662) and Charles's Law (1787) postulated the concept of 0K that was later supported by Avogadro's Law (1811) and statistical mechanics of L. Boltzmann $(1877,1884)$. The scholars recently see or expect to see evidence of temperature lower than $0 \mathrm{~K}$ [10], [11], [12], [13].

All gases ought to liquefy, solidify or become plasma at 0 $\mathrm{K}$, which is $273 \mathrm{~K}$ below the triples-point of pure water [1]. At triples-point of water, which is $273.16 \mathrm{~K}$ at $101.325 \mathrm{kPa}$, water may co-exist as ice to skate upon, melt to let creatures swim and evaporate to let clouds form. Gas-liquid-solid (GLS) phase of matter alters mainly with the change in temperature and pressure. Several laboratories have tabulated the straight as well as the exceptional cases of this transition.
The study of cryogenic temperature speaks more about gas phase rather than about liquid and solid phases. Gas loses gaseousness at $0 \mathrm{~K}$; so there is an expectation of "no gas" state at $0 \mathrm{~K}$ or gas-limit-temperature (GLT). The gas laws work without a fault principally because there is no gas below GLT. Logically, a "no liquid" state should exist below $0 \mathrm{~K}$ where all liquids solidify at liquid-limit-temperature (LLT). The same logic should allow a "no solid" state below LLT where, at solid-limit-temperature (SLT) all solids would let matter dissipate. SLT is the lowest limit of temperature that concerns matter and it occupies the emphatic point of consideration in this paper.

SLT is not a casual coextensive arrived unconcernedly while laying down an allegory of classes of temperature. Below SLT, the togetherness of time, energy, matter and probability remains. However, a vast storage of locked-upenergy that made and kept them all together in the world and the universe that humans know about, like pen-paper-ink, must find a way out from their bondage with matter. No one can know for sure what role heat may play in that situation 
and what other forms of energy might do. One can even theorise a concept of meta-energy $\in$ that heat, electricity, magnetism, attraction, momentum and other known manifestations of energy may own up as originator, and as argument of forms of energy that derive from $\in$, and those lower forms of energy shake off their mutual differences due to extreme coldness. Probably, Boltzmann also thought likewise and tried a hand at this.

Take $\Psi(\epsilon, \delta)$ or $\Psi(\delta)$ where $\delta$ or dromos ( $\delta \rho$ ó $\mu$ o $\zeta)$ or heat in Greek. Greek is a good language that attracts many nations. Take again $\Psi(\in, \eta)$ or $\Psi(\eta)$ where $\eta$ or ilectrismos

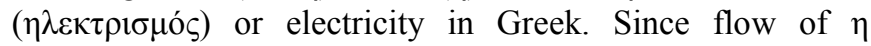
produces $\delta$ and thermo-couples produce $\eta, \delta$ and $\eta$ are not greatly distanced as energy and may belong to a common regime like $\Psi(\in)$. When humans get out of the doldrums of 0 $\mathrm{K}$, then they may find different forms of energy huddling nearer to $\in$. They huddle, get bigger as matters let go energy as cold becomes colder. That shows that the time or conscious-time [5] is cold-resistant and stays to measure the progress of coldness as matter dissipates. Conscious-time accompanies the change in phases from pre-GLT to GLT to LLT to SLT stages. Energy dissipates continually in conscious time as pre-GLT reaches the stage of SLT. Nearer to or at SLT, matter dissipates more predominantly. Again, since conscious time essentially is a finite linear series in composition [2], $\Psi(\delta)$ and $\Psi(\eta)$ may inter-relate, at least probabilistically, taking physics with them to reach a new vista.

In studying the properties of superconductivity of the solids and other issues of thermo-dynamics, all equations anchor at $0 \mathrm{~K}$; and at that temperature the entropy is supposedly, but erroneously, zero. Even when temperature as close to one millionth part of a centimetre away from $0 \mathrm{~K}$ was reached in 1997, there was the complacency that superconductivity of the conductors and non-conductors was to be investigated within the limit of $0 \mathrm{~K}$.

Heat energy is existential and multiplicity of its ranges contains matter, life or mind together or group-by-group. Similarly, electricity is existential and multiplicity of its ranges contains matter, life or mind together or group-bygroup.

\section{Limit of Low Temperature, Freeze Decay, Dissipation of Matter, Harvest of Cold Energy}

\subsection{Cold State}

Johannes Diederik van der Waals of Holland (1837-1923) had won Nobel Prize for Physics in 1910 for research on gaseous and liquid states of matter. He had proposed in 1873 what is now called the "van der Waals force". It relates to the understanding that the neutral molecules of gas, liquid and solids attract each other with a weak electric force. This force is proportional to the inverse of the seventh power of the distance between the centres of the atoms or molecules.
Atoms and molecules exist under the normal conditions of nature and they are the building blocks of matter for the human. The basic feature of the binding forces of atoms and molecules arise out of the uncertainty of quantum fluctuations in the distribution of the electrons that changes constantly as the electrons revolve "around" the nucleus in an atom (Rutherford, 1911). The process is internal to atom and proves experimentally correct every time. The molecules, composed of atoms, are independent entities. There is low probability that the molecules of gas meet one another or collide against the wall of the container (classical idea of gas pressure). At a low temperature, the mutual attraction between the molecules does not depend upon or arise from the random meeting of the molecules. The attraction more likely arises from van der Waals' Force. As molecule is not round like a sphere or football, atom is also not like a globe by shape. Kept inside a container, an atom may behave as per van der Waals' Force. In other words, any matter whether a molecule, atom, particle or sub-particle may have energybased attraction that is independent of gravity in the likeliness of the time-varying dipole.

The volume of a given weight of gas is 1,600 times greater than the volume of an equal weight of liquid (Encyclopaedia Britannica). When the temperature is kept above the "critical temperature" (Cagniard, 1822), then the compression of a gas from 1,600 units of volume to 1 unit of volume does not affect the phase of gaseousness - gas remains as compressed gas. On the other hand, if the temperature is lowered below the critical temperature, the gas turns abruptly liquid when compressed (Cagniard, case of $\mathrm{CO}_{2}$ ); thence the pressure remains unaltered until the whole of the gas turns liquid. Why this happens remains an unsolved problem of statistics and physics. In other words, at the critical volume or density, critical temperature and critical pressure, or at or above the "critical point" of fluid, liquid and its vaporous gas are the same; and below that temperature, GLS exists unfathomably.

The search for similar critical points had spread out, especially to the spheres of magnetism and electronics. Mathematics stated something like this: "If a function is defined for $\mathrm{n}$ dimensional manifold, its behaviour may be studied by introducing $\mathrm{n}$ coordinates in which the function becomes just a function of the same coordinates, then, such point or set of points is a critical point if its partial derivatives are zeros" [2-4]. The idea has application in finite calculus and study of arcs; however, it is doubtful if logic allows this mathematical concept of critical point to invade physics. On the other hand, the critical points obviously have the non-mathematical potential of replication for the change of state from liquid to solid at lower temperature. As suggested before in this essay, such changes are not yet authenticated for the simple reason that it involves the concept of temperature to go below $0 \mathrm{~K}$, which is not acceptable to physics; but should be. It is peculiar that statistics had accepted the truncated $0 \mathrm{~K}$ limit of temperature.

This hundred-year old study went out of focus. The special theory of relativity (STR) of A. Einstein surfaced in 1905 to remove the inconsistency of Newton's mechanics, Maxwell's 
equation of electromagnetism and earth's motion through luminiferous aether. Einstein had opposed the Boltzmann's "entropy equation". STR deals with motion nearing the speed of light. There was good fallout of consequences of STR. STR uses light. Light is energy, light is wave and light is particle. Light is dynamic. Light does not exist at present or in a static form. Light leaves all inertial systems behind. The laboratories cannot create or hold a light based system. All light-based systems are history. Laboratories have tried to prove that matter may move faster than light. In other words, light may not catch up with matter all the time. Therefore, a light-matter system cannot have an inertial mooring. An inertial or non-accelerating system does not exist.

Light-path is not the same as heat-path.

The present paper reminds that the study of cryogenics had preceded van der Waals discovery by about a century; and that study of cryogenics and the study of van der Waals are stuck. The effort should be to following the older track and attempting to finding temperature below $0 \mathrm{~K}$ empirically.

\subsection{Cold Force}

The similitude of gas and liquid influences the thoughts on the solid phase of matter. When the assembled molecules carry no net charge, "dipole moments", the van der Waals Force provides the crystal binding. An example is of diatomic molecule of hydrogen that is gas in room temperature, liquid at $41 \mathrm{~K}$ and solid at $20 \mathrm{~K}$. The molecular structure of hydrogen remains the same for its gaseous, liquid and solid phases and van der Waals Forces provide the crystal binding. Similarly, the aggregates of atoms, molecules or ions that remain as tiny parcels for short periods form "cluster". Clusters show effects on melting points of solid and electrical conductivity-like a cluster of a few atoms of mercury are insulators while the individual atoms of mercury conduct electricity. Importantly, why do the properties of clusters differ with the size of the clusters? There should be an external or selfgenerated force to do that.

There is a cut-off point in temperature below which the "frozen" metals show the properties of superconductivity. At temperatures below the transition temperature at which the logarithm of the electronic specific heats tends to decrease inversely as the superconductivity moves to a higher state, the distribution of energy levels available to the electrons in the superconductor mismatch for all conductors, and even for those that are non-conductors at normal temperature.

When (a) the temperature is lowered further to reach a temperature closer to $0 \mathrm{~K}$, (b) the matter is specific, like copper oxide-cuprate, and (c) the pressure is inconsequential; a second transition temperature is reached then the superconductivity of "no resistance" class sets in. The reason is assigned to "pairing of electrons". However, where did the resistance of matter to let electricity pass go? More questions naturally arise as whether this is the last of the transition temperatures that had been studied. (d) Could electric energy and heat energy lose their differences at low temperature? The mismatch of electronic excitation with thermal or other types of energy levels at $0 \mathrm{~K}$ should be many more. Considering that, (i) there must be a zero point energy, (ii) molecular movements are theoretically eliminated, and (iii) the electrons stay excited at least inside the molecule; there would be (iv) a left out of free, or paired, or grouped electrons that retained energy at $0 \mathrm{~K}$. This is because (v) it is not logical to assume that the process of super-superconductivity would terminate suddenly. It is possible that the electrons would assemble and (vi) self-generated power or nova-like burst of energy would result at another critical point of temperature. Thence, at another critical point of lower temperature, (vii) matter would dissipate into particles and neutrons into subparticles, and the particles and sub-particles would escape into the physicality of space.

Entropy is not "zero" at $0 \mathrm{~K}$. The construction of the third law of thermodynamics is statistical and there can be doubts like (viii) why a system should be at the ground state at $0 \mathrm{~K}$ with a logarithmic function of degeneracy (Boltzmann, 1877). This is, however, not the reason why Einstein opposed Boltzmann.

\subsection{Use of Older Literature}

Quantum theory remains unaffected by the existential theory of time [5]. However, there is another predicament. If the molecule has a zero-point energy, the kinetic energy of atoms carries a positive value at $0 \mathrm{~K}$. That provides the potential energy to the molecule by virtue of its known position at $0 \mathrm{~K}$. Neither the kinetic energy of the atoms nor the potential energy of the molecule can be there without associating conscious time with energy. The graph of energy and temperature on time may meet abscissa asymptotically; however, not when temperature is $0 \mathrm{~K}$ where energy exists and therefore, heat exists. It is obvious that $0 \mathrm{~K}$ should not be the lowest limit of temperature [6].

There is already an understanding that solids are not always molecules or atoms. Solid may be groups of electrons. White dwarf is hot because the electrons do not stay inert (uncertainty principle). The neutron stars are cold and get colder because they emit energy.

A digression is necessary. The force that keeps subparticles together in a particle is stronger than the force that keeps particles together. The force that keeps particles together in an atom is stronger than the force that keeps atoms in an element together. The force that keeps atoms in an element together is stronger than the force that keeps element in a molecule together. This "stronger than thou" of forces goes on and stops at gravity that is the weak force of cosmos. If atom or heavier build-ups are called "solid", then the temperature at which atom loses energy to let the particles go [7], is SLT or lower than SLT.

\subsection{Freeze Decay and Dissipation}

In case gravity goes on increasing, and the composite breaks half-and-half, and rounded, the split two-ness of the 
composite becomes about one-fourth of the original oneness of the rounded composite volume. Should the split be at one to nine ratios, the rounded splits retain three-fifth of the original rounded composition. Therefore, there is a good possibility that chipping away of the bits of material is more likely than an outright split of half-and-half or in other proportions [7]. The process would start, evidently, much before SLT. This is "Freeze Decay" [7, 8].

The general theory of time [7] reveals a new aspect of temperature and gravity. There are different and special absolute zero temperatures for gas, liquid and solid. When "absolute zero temperature of solid" is nearly reached and the internal "binding" energy of matter is dissipated below a critical limit, there are conditions that the force of gravity may be higher than the force that keeps the particles and the sub-particles of solid together. At that stage "dissipation" of matter may start.

At very low temperature, matter turns to dissolute particles just as it does at very high temperature and pressure.

Matter, as we know it, exists between two critical points of temperature, just as life does. Surprisingly, very old world literature supports such a view [9].

\section{Conclusion}

The lowest limit of temperature is lower than $0 \mathrm{~K}$. It is unlikely that entropy can attain a zero value at $0 \mathrm{~K}$.

There are critical points at a very low temperature where the molecules, atoms and neutrons lose energy to such extent that they cannot keep the constituent electrons and other particles together. Matter decays. When that happens, the electrons, other particles and sub-particles that compose the molecule, atom and neutron dance away into the space surrounding the molecule, atom and neutron, possibly, after a burst of energy.

Human should harvest this burst of energy. It may be possible to regulate cooling. Empirical discovery of temperature lower than $0 \mathrm{~K}$ has great importance.

Heat dissipates in quantum in motion during an "instant" or a "moment" of conscious time. That is a good foundation to go ahead with the study [5].

\section{Acknowledgement}

The author acknowledges M/S Knowledge Isotopes Pvt. Ltd for writing support. (http://www.knowledgeisotopes.com)

\section{References}

[1] "The American Heritage, Science Dictionary, Houghton Muffin Company," triples point, 2015.

[2] Stewert, and James, Calculus, Early Transcedentals, 6th ed., 2008.

[3] R. Larson, and B. H. Bruce, Calculus, 9th ed., 2009.

[4] R. A. Adam, and C. Essex, Calculus, A Complete Course, 2009.

[5] J. K. Barthakur, "Existential Theory of Time, Special Issue: Physics of Time: Theory and Experiment," American Journal of Modern Physics, Vol. 4, pp. 19-25, 2015.

[6] J. K. Barthakur, "Limit of Low Temperature, Freeze Decay," Essays in the Year of Physics, 2005.

[7] J. K. Barthakur, "The Limitation in the Concept of Space Time Continuum", Indian Science Congress Association, The 89th Congress held in Lucknow, 2002.

[8] J. K. Barthakur, Theory of Time, Indian Philosophical Quarterly, 1995.

[9] J. K. Barthakur, "Black Hole Should Not Exist", Indian Science Congress Association, The 90th Congress held in Bangaluru, 2003.

[10] S. Braun et al. Negative Absolute Temperature for Motional Degrees of Freedom,Science 339, 52-55 (2013).

[11] A. Rapp, S.Mandt, and A.Rosch, Equilibration Rates and Negative Absolute Temperatures for Ultracold Atoms in Optical Lattices, Phys. Rev. Lett. 105, 220405 (2010).

[12] S. Mandt, A. Rapp, and A. Rosch, Interacting Fermionic Atoms in Optical Lattices Diffuse Symmetrically Upwards and Downwards in a Gravitational Potential, Phys. Rev. Lett. 106, 250602 (2011).

[13] P. Medley, D. M. Weld, H. Miyake, D. E. Pritchard, and W.Ketterle, Spin Gradient Demagnetization Cooling of Ultracold Atoms, Phys. Rev. Lett. 106, 195301 (2011). 